\title{
TWO METHODS FOR CONSISTENCY-DRIVEN APPROXIMATION OF A PAIRWISE COMPARISON MATRIX
}

\author{
Dmitriy Borodin* \\ Production Information Systems Lab \\ Univers ity College Ghent \\ Ghent, Belgium \\ E-mail: dmitriy.borodin@,hogent.be \\ Victor Gorelik \\ Dorodnicyn Computing Center of Russian Academy of Sciences \\ Moscow, Russia \\ E-mail: vgor16@mail.ru \\ Wim De Bruyn \\ Production Information Systems Lab \\ University College Ghent \\ Ghent, Belgium \\ E-mail: wim.debruyn@,hogent.be \\ Bert Van Vreckem \\ Production Information Systems Lab \\ Univers ity College Ghent \\ Ghent, Belgium \\ E-mail: bert.vanvreckem $a$,hogent.be
}

\begin{abstract}
Pairwise comparisons are central in mathematics for the measurement of intangible factors, as proved in the Analytical Hierarchy/Network Process theory. In order to recover the scale for decision making, it is necessary to solve the corresponding eigenvalue problem, which brings us to the pairwise comparison matrix inconsistency.

The paper proposes two methods for approximating an inconsistent reciprocal matrix by a consistent one. In the first method, the approximation problem is formulated and solved using the Lagrange multipliers method. In the second method, the problem is formu lated as a correction problem for an inconsistent set of linear equations with a spare structure.
\end{abstract}

Keywords: pairw ise comparison, approximation, correction, inconsistency

\section{Introduction}

The analytical hierarchy process (AHP) (Saaty, 2008) provides us with an effective method to present human evaluations numerically.

\footnotetext{
* Corresponding author
} 
A very important role in this method belongs to a pairwise comparison matrix. It represents an expert's judgments for this or that object or event.

$$
A=\left(a_{i j}\right)_{n \times n}=\left(\begin{array}{cccc}
a_{11} & a_{12} & \cdot & a_{1 n} \\
a_{21} & a_{22} & \cdot & a_{2 n} \\
\cdot & \cdot & \cdot & \cdot \\
a_{n 1} & a_{n 2} & \cdot & a_{n n}
\end{array}\right)
$$

Each element $a_{i j}$ of the matrix shows the importance of the $A_{i}$ object comparing to $A_{j}$. The judgments are given according to a special scale.

Once the matrix has been filled in, it is necessary to evaluate the importance of each criterion. The following condition must be true to ensure the existence of the weight coefficients: $a_{i j} a_{j k}=a_{i k}$, for all $i, j, k$ (particularly, $a_{i j}=\frac{1}{a_{j i}}$ ). The proof of this statement can be found in (Saaty, 2008).

In this case the problem is in calculating the eigenvector of the matrix related to its maximum eigenvalue. As it is proved, a positive antisymmetric matrix is consistent if and only if $\lambda_{\max }=n$, where $\lambda_{\max }$ is the maximum eigenvalue of the matrix and $n$ is its dimension. Only in this case the eigenvector gives the precise values of the criteria weights.

But usually the pairwise comparison matrix is inconsistent, i.e. $a_{i j} a_{j k}=a_{i k}, i \neq j$ is true not for all $i, j, k \in\{1,2, \ldots, r\}$. Saaty (Saaty, 2008) suggested using a consistency ratio (CR) to decide whether the approximate calculation of the matrix eigenvector and eigenvalues can be accepted as criteria weights or not. And if the value of $\mathrm{CR}$ is not acceptable, it is necessary to repeat the decision-making process, i.e. fill in the pairwise comparison matrix again.

There are different methods developed either to prevent such incons istency or to correct it.

The first group of methods includes such methods as the simplified decision-making procedure (Nogin, 2004), interval estimations (Podinovsky, 2007).

The second group proposes the methods for correcting the elements of an inconsistent pairwise comparison matrix follow ing the objective that the corrected matrix is as close as possible to the original one. The original idea of the current paper is similar to (Dopazo and González-Pachón, 2003), but the approximation methods are different and were inspired by the authors' work for solving inconsistent sets of linear equations. The authors use term "correction" both for the matrix and for its elements.

\section{Two consistency-driven approximation methods}

From the matrix we have an inconsistent set of equations. Let's define the problem of correcting the pairwise comparis on matrix:

$$
\begin{gathered}
\|\mathrm{H}\|^{2} \rightarrow \min \text {, so that }(\mathrm{A}+\mathrm{H}) \text { is consistent, } \\
\text { i.e. }\left(a_{i j}+h_{i j}\right)\left(a_{j k}+h_{j k}\right)=a_{i k}+h_{i k} \text {, where } h_{i j} \text { belongs to } \mathrm{H} \text {. }
\end{gathered}
$$

Below we explain two approaches to solving this correction problem. The quadratic criterion is used as the approximation criterion.

\subsection{Solving the problem using the method of Lagrange multipliers.}

The problem of correcting pairw ise comparison matrix elements can be expressed as follows:

$$
\sum_{i=1}^{r-1} \sum_{j>i} h^{2} \rightarrow \min ,\left(a_{i j}+h_{i j}\right)\left(a_{j k}+h_{j k}\right)=a_{i k}+h_{i k}
$$

By applying the method of Lagrange multipliers, the following expressions are obtained: 


$$
\begin{gathered}
L=\sum_{i=1}^{r-1} \sum_{j>i} h_{i j}^{2}+\sum_{i, j, k} \lambda_{i j k}\left(a_{i j} h_{j k}+a_{j k} h_{i j}-h_{i k}+h_{i j} h_{j k}\right) \\
\frac{\mathbb{\Phi} L}{\mathbb{\Upsilon} h_{i j}}=2 h_{i j}+\lambda_{i j k} a_{j k}-\lambda_{i j k} h_{j k}=0
\end{gathered}
$$

It is difficult to find the solution of problem (1) using the method of Lagrange, but the values of $h_{i j}, h_{j k}$ have the second order of vanishing and, by considering them as zero, the problem is reduced to the quadratic programming problem, which is solvable.

2.2 Solving the correction problem by correcting the inconsistent set of linear equations with the spare structure.

By expressing the coefficients of the pairwise comparison matrix using their definition through the criteria weights, we define the following problem:

$$
\begin{aligned}
& \sum_{i=1}^{r-1} \sum_{j>i} h^{2} \rightarrow \min , \\
& W_{i}=\left(a_{i j}+h_{i j}\right) W_{j}, \forall i=\overline{1, r-1}, j>i, \\
& \sum W_{i}=1 .
\end{aligned}
$$

Thus, we have the problem of correcting a set of linear equations with the spare structure:

$$
\left(\begin{array}{cccccc}
1 & -a_{12} & 0 & \ldots & \ldots & 0 \\
1 & 0 & -a_{13} \ldots & \ldots & 0 \\
\ldots \ldots \ldots \ldots \ldots \ldots \ldots \ldots \ldots . \\
0 & 1 & -a_{23} \ldots & \ldots & 0 \\
0 & 1 & 0 & -a_{24} \ldots & 0 \\
\ldots & \ldots \ldots \ldots \ldots \ldots \ldots \ldots . \\
0 & 0 & \ldots \ldots \ldots \ldots & - \\
1 & 1 & \ldots \ldots \ldots \ldots \ldots \ldots \ldots & 1
\end{array}\right)\left(\begin{array}{l}
x_{1} \\
x_{2} \\
x_{r-1, r} \\
x_{(r-1) / 2)+1}
\end{array}\right)=\left(\begin{array}{l}
0_{r(r-1) / 2} \\
1
\end{array}\right)=e .
$$

It is necessary to find the correction matrix $\mathrm{H}^{*}$ so that the system $\left(\mathrm{A}+\mathrm{H}^{*}\right) \mathrm{x}=\mathrm{e}$ is consistent and the following condition is true:

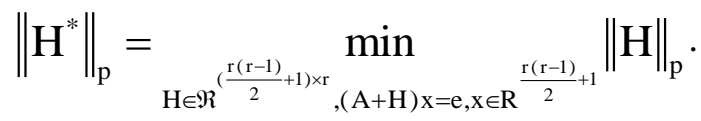

The matrix of correction $\mathrm{H}$ has the following structure:

$$
\mathrm{H}=\left(\begin{array}{cccccc}
0 & -\mathrm{h}_{12} & 0 & \ldots & \ldots & 0 \\
0 & 0 & -\mathrm{h}_{13} \ldots & \ldots & 0 \\
\ldots \ldots \ldots \ldots \ldots \ldots \ldots \ldots \ldots & \ldots \ldots \ldots \\
0 & 0 & -\mathrm{h}_{23} \ldots & \ldots & 0 \\
0 & 0 & 0 & -\mathrm{h}_{24} \ldots & 0 \\
\ldots \ldots \ldots \ldots \ldots \ldots \ldots \ldots \ldots \ldots \ldots & \ldots \ldots \ldots \ldots \\
0 & 0 & \ldots \ldots \ldots \ldots . .0 & -\mathrm{h}_{\mathrm{r}-1, \mathrm{r}} \\
0 & 0 & \ldots \ldots \ldots \ldots \ldots \ldots \ldots & 0
\end{array}\right) .
$$

The following vector is related to Hunambiguously: 


$$
\alpha=\left(-\mathrm{h}_{12},-\mathrm{h}_{13}, \ldots .,-\mathrm{h}_{1 \mathrm{r}},-\mathrm{h}_{23}, \ldots,-\mathrm{h}_{2 \mathrm{r}}, \ldots \ldots,-\mathrm{h}_{\mathrm{r}-1, \mathrm{r}}\right) \in \mathrm{R}^{\frac{\mathrm{r}(\mathrm{r}-1)}{2}}
$$

Now we define the non-conditional optimization problem:

$$
\left\|\left[\begin{array}{c}
\mathrm{r}(\alpha, \mathrm{x}) \\
\alpha
\end{array}\right]\right\|_{\mathrm{p}} \rightarrow \min _{\alpha, \mathrm{x}}
$$

Then we link vector $\mathrm{x}$ with matrix $\aleph(\mathrm{x})$ :

$$
\aleph(x)=\left\{x_{r s}:\left\{\begin{array}{l}
x_{r s}=x_{j_{1}}, \text { if } r=i_{1}, s=1,1=1, \ldots, k, i_{1} \in I_{A}, j_{1} \in J_{A} \\
x_{r s}=0, \text { in other cases }
\end{array}\right\}\right.
$$

where sets $\mathrm{I}_{\mathrm{A}}$ and $\mathrm{J}_{\mathrm{A}}$ are expressed as follows:

$$
\begin{aligned}
& \mathrm{I}_{\mathrm{A}}=\left\{\mathrm{i}:(\mathrm{i}, \mathrm{j}) \in \mathrm{K} \backslash \mathrm{K}_{0}\right\}=\left\{\mathrm{i}_{1}, \ldots, \mathrm{i}_{\mathrm{k}}\right\}=\left\{\mathrm{i}_{1}: \mathrm{l}=1, \ldots, \mathrm{k}\right\}, \\
& \mathrm{J}_{\mathrm{A}}=\left\{\mathrm{j}:(\mathrm{i}, \mathrm{j}) \in \mathrm{K} \backslash \mathrm{K}_{0}\right\}=\left\{\mathrm{j}_{1}, \ldots, \mathrm{j}_{\mathrm{k}}\right\}=\left\{\mathrm{j}_{1}: \mathrm{l}=1, \ldots, \mathrm{k}\right\} .
\end{aligned}
$$

After that we linearize (3) considering the spare structure.

We linearly add $\Delta \alpha$ and $\Delta \mathrm{x}$ to vectors $\alpha$ and $\mathrm{x}$ respectively. Thus, we consider $\mathrm{r}(\alpha+\Delta \alpha, \mathrm{x}+\Delta \mathrm{x})$. As it is proved in (Zoltoeva, 2006), the following identical equation is true:

$$
\mathrm{H}(\Delta \alpha) \mathrm{x}=\aleph(\mathrm{x}) \Delta \alpha .
$$

As $\mathrm{H}(\Delta \alpha) \Delta \mathrm{x}$ is of the second order of vanishing, we consider it as zero; then we get the following expression:

$$
\mathrm{r}(\alpha+\Delta \alpha, \mathrm{x}+\Delta \mathrm{x})=\mathrm{r}(\alpha, \mathrm{x})-\aleph(\mathrm{x}) \Delta \alpha-(\mathrm{A}+\mathrm{H}(\alpha)) \Delta \mathrm{x} .
$$

Now we solve problem (3) using the total-least norm algorithm (TLN-algorithm):

Step 0. (initialization). Set vectors $\alpha \in \mathrm{R}^{\mathrm{k}}$ and $\mathrm{x} \in \mathrm{R}^{\mathrm{n}}, \mathrm{k}=\operatorname{dim}\left(\mathrm{K} \backslash \mathrm{K}_{0}\right)$.

Step i. (iteration). Solve the following auxiliary problem:

$$
\left\|\left[\begin{array}{cc}
\aleph(\mathrm{x}) & \mathrm{A}+\mathrm{H}(\alpha) \\
1_{\mathrm{\kappa}} & 0_{\mathrm{n}}
\end{array}\right]\left[\begin{array}{c}
\Delta \alpha \\
\Delta \mathrm{x}
\end{array}\right]+\left[\begin{array}{c}
-\mathrm{r}(\alpha, \mathrm{x}) \\
\alpha
\end{array}\right]\right\|_{\mathrm{p}} \rightarrow \min _{\Delta \alpha, \Delta \mathrm{x}}
$$

where $1_{\mathrm{k}}$ and $0_{\mathrm{n}}$ are vectors consisting of $0 \mathrm{~s}$ and $1 \mathrm{~s}$ and having the corresponding lengths.

If $\left\|\left[\begin{array}{l}\Delta \alpha \\ \Delta \mathrm{x}\end{array}\right]\right\|_{\infty} \leq \varepsilon$, then stop, otherwise set $\alpha=\alpha+\Delta \alpha, \mathrm{x}=\mathrm{x}+\Delta \mathrm{x}$ and continue to step $\mathrm{i}$.

In each step of the proposed TLN-algorithm the auxiliary problem (4) can be solved using different methods depending on the value of $p$. If $p=2$, then the problem is reduced to solving a consistent set of algebraic linear equations using the least squares method, which, in its turn, can be solved either by applying the pseudo inverse to the following matrix

$$
\mathrm{M}=\left[\begin{array}{cc}
\aleph(\mathrm{x}) & \mathrm{A}+\mathrm{H}(\alpha) \\
1_{\mathrm{K}} & 0_{\mathrm{n}}
\end{array}\right]
$$

or by factorizing this matrix as a multiplication of the orthogonal $(\mathrm{Q})$ and upper triangle $(\mathrm{R})$ matrices, i.e. by the so-called QR-factorization (Horn and Johnson, 1985). If $p=\infty$, subproblem (4) is reduced to the following linear programming problem:

$$
\mathrm{u} \rightarrow \min _{\mathrm{u}, \Delta \alpha, \Delta \mathrm{x}}
$$




$$
\left\{\begin{array}{l}
\mathrm{u} \geq \aleph(\mathrm{x}) \Delta \alpha+(\mathrm{A}+\mathrm{H}(\alpha)) \Delta \mathrm{x}-\mathrm{r}(\alpha, \mathrm{x}), \\
\mathrm{u} \geq-\aleph(\mathrm{x}) \Delta \alpha-(\mathrm{A}+\mathrm{H}(\alpha)) \Delta \mathrm{x}+\mathrm{r}(\alpha, \mathrm{x}), \\
\mathrm{u} \geq \alpha+\Delta \alpha, \\
\mathrm{u} \geq-\alpha-\Delta \alpha .
\end{array}\right.
$$

\section{Conclusions and future work}

The paper proposed two methods for correcting the elements of an inconsistent pairwise comparison matrix in order to find a consistent matrix which is "as close as possible" (Dopazo and González-Pachón, 2003 ) to the original one. The described approaches are particular cases of a more general research that deals with the approximation of inconsistent sets of linear equations; it was shown how to reduce the problem of the pairwise comparison matrix approximation to the problem of correcting the inconsistent linear set with a certain structure.

Next steps of this research include generalization of the approaches, detailed numerical experiments and analysis, linking the current work and the research of Dr. Esther Dopazo and her colleagues (Dopazo and González-Pachón, 2003).

\section{Acknowledgments}

The paper is dedicated to the memory of our beloved colleague and friend Dr. Irina A. Zoltoyeva.

\section{REFERENCES}

Dopazo, E., \& González-Pachón, J. (2003). Consistency-driven approximation of a pairwise comparison matrix. Kybernetika, 39(5), 561-568.

Horn, R. A. \& Johnson, C.R. (1985). Matrix Analysis. Cambridge University Press.

Nogin, V. (2004). A simplified method of AHP based on the non-linear criteria convolution. Journal of Computational Mathematics and Mathematical Physics, 44(7), 1259-1268 (in Russian).

Podinovski, V. (2007). Interval articulation of superiority and precise elicitation of priorities. European Journal of Operational Research, 180(1), 406-417.

Saaty, T. L. (2008). Relative Measurement and Its Generalization in Decision Making. Why Pairwise Comparisons are Central in Mathematics for the Measurement of Intangible Factors. The Analytic Hierarchy/Network Process. Statistics and Operations Research, 102(2), 251-318.

Zoltoyeva, I.A. (2006). Data correction methods for formalization and solution of multicriteria optimization problems. Ph.D. thesis (in Russian). 